\title{
Remote Collaborative Multi-User Informal Learning Experiences: Design and Evaluation.
}

\author{
Ernesto Arroyo ${ }^{1}$, Valeria Righi ${ }^{1}$, Roger Tarrago $^{1}$, Patricia Santos ${ }^{1}$, Davinia \\ Hernandez-Leo ${ }^{1}$, Josep Blat ${ }^{1}$ \\ ${ }^{1}$ GTI, ICT Department, Universitat Pompeu Fabra, Roc Boronat 138, \\ 08018 Barcelona, Spain \\ \{ernesto.arroyo, valeria.righi, roger.tarrago, patricia.santos, \\ davinia.hernandez, josep.blat \}@upf.edu
}

\begin{abstract}
This paper presents a customizable system used to develop a collaborative multi-user problem solving game. It addresses the increasing demand for appealing informal learning experiences in museum-like settings. The system facilitates remote collaboration by allowing groups of learners to communicate through a videoconferencing system and by allowing them to simultaneously interact through a shared multi-touch interactive surface. A user study with 20 user groups indicates that the game facilitates collaboration between local and remote groups of learners. The videoconference and multitouch surface acted as communication channels, attracted students' interest, facilitated engagement, and promoted inter- and intra-group collaborationfavoring intra-group collaboration. Our findings suggest that augmenting videoconferencing systems with a shared multitouch space offers new possibilities and scenarios for remote collaborative environments and collaborative learning.
\end{abstract}

Keywords: Computer supported collaborative learning, ubiquitous learning, informal learning, serious games, social awareness

\section{Introduction}

This paper presents a customizable system designed to support collaborative multiuser problem solving in remote environments. It addresses the need for collaborative informal learning experiences in museum-like environments. The system combines multi-touch interaction and videoconferencing to support remote multi-user collaborative learning experiences. The system has been used to develop a serious game where groups of remote museum visitors interact with each other. During the game students put in practice their collaborative, communication and negotiation skills. They also share their knowledge acquired in the museum with their gamemates. The interactive experience is capable of engaging students in collaborative problem solving activities. It takes advantage of students' curiosity and attractiveness to new technologies to meet the increasing demand for appealing informal learning experiences [18] for use in museum-like settings. 
The system has been evaluated with 39 university first-year students in a controlled environment. The results indicate that the system promotes collaboration and communication among participants. The appeal of the multi-touch surface attracted students, facilitating engagement, and inter- and intra-group collaboration. Participants were more inclined to collaborate with co-located partners and provided feedback related to the screen setup for facilitating the ergonomics of the communication through videoconference.

\section{Background}

Moore (1989) proposed three main types of interaction that can be supported by synchronous interactive technology: the instructor-learner interaction, learner-learner interaction and learner-content interaction [14]. Several online and desktop-based systems and applications have been developed to support the three types of interaction, allowing simultaneous collaboration between multiple learners (remote or co-located). These systems have proved useful in facilitating collaboration and provision of instant formative feedback and improving the cognitive proximity and identity decoupling of students [19]. Some of these collaborative systems use readily available commercial hardware, such as the Microsoft Multipoint ${ }^{1}$ project, which integrates up to 25 mice and allows students in a classroom to simultaneously interact and work together on a single desktop computer. Each student is equipped with a computer mouse and Multipoint software assigns a unique mouse pointer to each student, giving him or her equal opportunity to participate in coordinated, collaborative, or cooperative activities through single display groupware [17]. Other collaborative systems include online tools to allow learners to participate using a personal computer. For instance, GroupScribbles ${ }^{2}$ is an application for realizing collaborative learning activities especially designed for primary school students [19]. Students and teachers interact with the system from their own computers and use several artifacts (such as adhesive notes, bulletin boards, whiteboards, stickers) to share their knowledge. They can create their own artifacts in their private space and share them in the public space. As [12] identified, by using GroupScribles students have the opportunity of playing active roles in their learning, they can interact with their peers and teachers. GroupScribles has been designed to allow multiple users interacting with the system at the same time; however it does not support a group of students interacting with the same computer at the same time.

Touch and Tangible User Interfaces (TUI) have already proved useful in supporting interaction between multiple learners. In touch and TUI learners interact and manipulate digital information through touch and physical objects [5]. Interacting with tabletops, smartboards, multitouch screens and tangible artifacts, facilitates simultaneous interaction and provides new opportunities to envisage innovative learning scenarios: interactive tables might support participation balance in $\mathrm{f} 2 \mathrm{f}$

\footnotetext{
${ }^{1} \mathrm{http}: / /$ www.microsoft.com/windows/multipoint/

${ }^{2}$ http://groupscribbles.sri.com
} 
collaborative learning [3], multi-touch whiteboards might facilitate group learning [10], and tangible artifacts might be used to solve design problems in vocational education [8].

Videoconferencing systems can support collaborative learning activities at a distance6]. They offer a direct communication channel, allowing students to visualize each other and to freely voice their ideas and opinions, thus facilitating an effective remote collaboration experience [11]. Online collaborative systems incorporate videoconferencing and also provide the means for sharing a virtual desktop or for exchanging digital information, such as pictures, presentations, documents, etc. Examples of existing commercial products include flashmeeting ${ }^{3}$, wimba ${ }^{4}$ and Adobe Connect $^{5}$. Although video conferencing systems offer several advantages for remote learning, their use seldom goes beyond video calls in informal educational settings involving small to medium sized groups [20] [2]. This is not only due to their excessive installation costs and complex setup (for specialized equipment), but mainly to their lack of support for collaboration on complex scenarios that go beyond document and desktop sharing. For instance, when dealing with mixed settings with co-located and remote groups of learners, neither video-conferencing nor online collaboration systems support interaction within the local group. On one hand videoconferencing provides f2f (or group2group) communication, but restricts simultaneous participation and interaction. Furthermore, typical videoconferencing systems often force users to take-turns when working in groups by signaling their intent to participate and waiting until allowed to intervene. On the other hand online collaborative applications and single display-display groupware support simultaneous interaction over a shared space, but lack (f2f) natural communication between remote or local learners.

Augmenting videoconferencing systems with multi-touch interaction allows multiple learners to interact simultaneously over a tangible shared space, to maintain verbal communication, and to share non-verbal cues through an additional visual link [16]. This offers new possibilities and scenarios for remote collaborative environments and collaborative learning. For instance, in galleries or museum-like environments, visitors may want to collaborate in order to share their views, knowledge and interpretation of the elements exhibited. The social interactions can lead to learning outcomes, and together with the multi-touch experience engage the participants that might be strangers otherwise [25]. New interactive learning experiences can draw museum visitors and motivate students to further explore an exhibition during a school visit. Moreover, museums in different locations can be complementary to each other and the learning experienced by visitors in one museum could enrich the learning of visitors in the other.

\footnotetext{
${ }^{3}$ Flashmeeting Website, http://flashmeeting.e2bn.net

${ }^{4}$ Wimba Website, http://www.wimba.com/products/wimba_classroom

${ }^{5}$ Adobe Connect Website, http://www.adobe.com/products/adobeconnect.html
} 


\section{A Remote Collaborative Multi-user Learning Experience}

This section describes the design criteria used to fulfill the main collaboration and communication goals. We illustrate the design criteria by the development of a concrete experience involving two remote museums sharing a common installation. The goal of this experience is to facilitate collaborative problem solving while working in remote environments. The project also seeks to support one-to-one and many-to-many communication to allow more than one learner to participate and interact in informal learning experiences between remote and co-located learners. We describe how the system features and design considerations attempt to accomplish the communication, collaboration and interaction goals.

\subsubsection{Use Case and Scenario}

This project was developed within the context of two Spanish museums that exhibited an art collection simultaneously in Figueres and Barcelona cities. The common exhibition was titled "La col·lecció de col·leccions de Guy Selz" (The collection of collections of Guy Selz). It included random and hectic selection of objects from around the world that were collected by Guy Selz through his life travels [9]. Some objects included anecdotes and descriptions about their origin, uses, and importance. Each museum hosted an un-categorized sample from the collection with the intention to allow visitors to come up with their own categories. The unique characteristics of this use-case directed the application to become an additional channel for museum visitors to explore the exhibition and increase the sense of connectedness between two spaces. All the while, allowing museum visitors to interact and collaborate. The Toy Museum of Catalonia requested for the experience to be a game (one major design constrain) as they receive frequent guided school visits and students had expressed their interest in playing while at the museum (expected from a toy museum). Thus, during a coordinated activity in both museums, groups of students visiting the Arts Santa Monica museum in Barcelona and the Toy Museum of Catalonia in Figueres are invited to play a game with other groups of students also visiting a museum in a different city. The game is related to objects they have observed and experienced during their museum visit.

\subsubsection{Flexibility}

After having conducted interviews and participative workshops with cultural organizations, museum curators, technology providers and interaction designers, it was clear that no single interactive learning experience would fit all of the stakeholder's needs. Although all of them agreed that incorporating multi-touch surfaces and videoconferencing could enhance the visitor and learner experience, they did not agree on a single scenario, as there seemed to be endless alternatives. Some of the envisioned scenarios included treasure hunting activities, collaborative puzzles, interactive videoconferencing and performances, collaborative music composition, collaborative tagging, expertise sharing, collaboratively exploring high definitions images, among others. From this experience we realized that it was necessary to develop an agile flexible system to provide the means for enacting different types of interactive learning games and experiences. The system uses an XML scheme to 
describe the user flow and the behavior of each element of the interface. In this way, it is enough to change few attributes and interface files to obtain another game with a different interface while maintaining the same logic.

\subsubsection{Educational Games}

Part of the design criteria has been based on the motivational generalizations and design principles proposed by [18]. First of all the game has been designed to provide formative feedback to help students to acquire the necessary expertise that they need to learn about the contents of the museum. Feedback with information about the pieces selected during the game and hints to find the correct solution are provided to the students. In addition the tasks have been designed in order that students feel confident and competent to solve them without many difficulties. Students interact with different types of artifacts and the game provides three levels in which students perform different tasks. All the tasks have been designed to stimulate students' motivation but also to provide learning content that can be meaningful and interesting for them.

\subsubsection{Collaboration}

Inter- and Intra-Group Collaboration

The effectiveness of communication and collaboration can be enhanced if group's activities are coordinated [6]. Thus the primary goal is to facilitate collaborative activities within a group of co-located students, while also facilitating remote collaboration and coordination between groups of students in different locations. The experience has been designed to facilitate intra-group and inter-group collaboration by incorporating focused collaborative problem-solving tasks that require collaborating with other participants and at the same time, they provide opportunities to attain social and academic goals [4][18].

Intra-group collaboration refers to discussion and interaction elicited to solve a problem task, in which co-located members are involved. At the other hand, intergroup collaboration refers to problem solving activities that involve members belonging to remote groups. In order to facilitate intra- and inter- group collaboration, the system provides students with a shared workspace where simultaneous and multiuser interactions are allowed for both co-located and remote participants. Table 1 describes the elements that have been specifically incorporated into the game to reinforce collaboration and discussion among remote groups: 1) students must complete problem-solving tasks and perform operations together with the remote partners in order to progress; 2) students can simultaneously work in different parts of the task both individually or collaboratively; 3 ) students must focus and provide help on a task that should be completed by the remote group.

Physical and Verbal collaboration

In collaborative environments individual participation can take place both through verbal communication and through physical actions [7]. Our system supports both physical and verbal intra- and inter-group communication and collaboration. It 
provides a videoconference channel for verbal communication and a shared virtual space for physical communication and collaboration.

Physical inter-group and intra-group collaboration is supported by simultaneous interactions with a multi-touch surface that allows more than one users to interact at the same time. Students can interact at the same time with the game interface elements among co-located (intra-group) and remote (inter-group) learners over a shared virtual space. On the shared virtual space deictic gestures are possible thanks to visual elements that provide awareness of the remote actions. Multi-touch screen on both ends facilitate inter- and intra-group students to have the same opportunities to collaborate and interact with the artifacts in order to solve problems together. A videoconference displayed on a vertical screen supports verbal inter-group collaboration, allowing remote participants to talk and discuss about game tasks.

\subsubsection{Equity of Collaboration}

Simultaneous interaction can prevent turns-taking behavior among participants and at the same time can promote equitable interaction Stanton 2002); however it can also result in parallel working and distribution of tasks among users [21]. In a remote environment parallel working and taking-turn behavior might constrain reciprocity and communication, therefore limiting collaboration among remote members. We adopted several design criteria to avoid limiting collaboration and promote an equal participation among remote partners. A multi-touch table allows for direct manipulation of digital content while at the same time allows for multi-user interaction. Several studies about coordination in tabletops settings have demonstrated that distributed tabletop interaction might be influenced by workspace configuration, leading to territoriality behavior and unequal participation among users [23]. We designed the virtual space with the objective of allowing one or more remote learners to interact simultaneously over a shared collaborative workspace. The system uses a "what you see is what I see" interaction metaphor [22] in which all participants share the same visual perspective of the shared workspace (same-side configuration). This configuration allows both remote groups to have equal opportunities for interaction, allowing them to make contributions for solving the problem and avoiding territoriality division of the surface [23]. Moreover, in order to facilitate an equal participation among co-located users, items of the interface were equally distributed on the whole surface.

\subsubsection{Communication and Awareness}

An important issue when designing remote collaborative environments is to provide a common channel for communication. This channel should facilitate emerging conversations and allow users to be aware of the actions of their remote partner [6]. The system includes a continuous videoconference link that promotes verbal communication and awareness between remote groups of students. The aim is that groups will use the videoconference to coordinate their actions verbally over the videoconference link. The system also supports remote awareness by using several visual cues and elements on the shared workspace that help learners understand the actions of the remote partners, providing common ground for deictic gestures that 
support communication. The system uses visual pointers of the finger in order to reduce interference within the space produced by multiple participants interacting simultaneously [24]. When user touches the screen a colored circle is "painted" under his finger (each location is represented by specific color). In this way, the actions of each student are immediately visualized on the screen of the remote location: when students move an object in one location, remote students will also see the same object moving marked with the color of the remote group.

\section{Prototype Description}

Two stations placed in two remote locations compose the system. Each station includes two 32' screens: one horizontal multi-touch screen, and a vertical screen (Fig. 1). The horizontal screen displays content related to the exhibition that can be seen and accessed from both stations. The vertical screen displays a video feed from each location and complementary information related to the learning experience (instructions, status, hints, and support material).

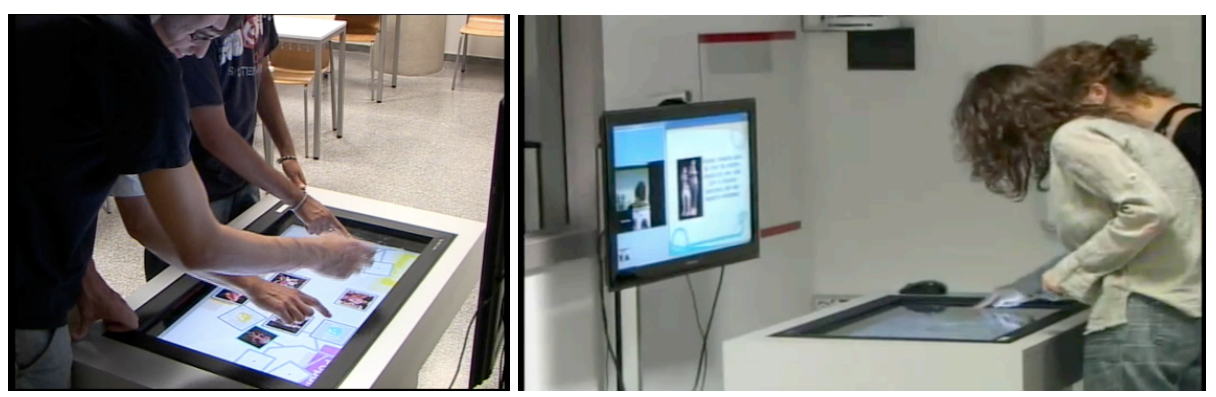

Fig. 1. The system includes two remotely located stations. Right: Participants interact on a horizontal surface while sharing the same workspace with remote partners. A vertical screen shows a video feed facilitating communication between the two locations. Left: Simultaneous co-located participants categorizing objects by country of origin and by functionality.

\subsection{Game Levels}

Collaborative tasks are introduced in stages in order to guide and facilitate discovering and exploring the educational material. The game is divided into three sequential stages and requires at least one participant on each remote location. Each stage starts with a brief description of the rules on the vertical screen. Coherent interaction throughout the different stages of the game facilitates transitioning between stages. In stage zero, or at the start of the game, users select the number of participants on each remote location-- from one, up to three players and cannot be changed during the game. The system uses the number of participants to control the numbers of fingers required to collaboratively select pieces in the following stages. The first stage presents information about the elements in the game and provides useful information for completing the remainder stages. This stage promotes 
negotiation and collaborative decision-making by requiring participants to coordinate their interaction and cooperatively select an item (cooperative manipulation [13]). The other two stages require participants to group items with respect to geographical or functional categories. In this phase of the experience all participants can simultaneously interact on the surface moving and placing objects on proper placeholders. These stages also include "special items" that have been designed to encourage cooperation and information sharing between remote participants. Table 1 summarizes the main features of the game and their relationship to the type of collaborative behavior elicited.

Table 1. Game stages and their relationship to collaboration objectives.

\begin{tabular}{|c|c|c|c|c|}
\hline Stage & $\begin{array}{l}\text { Game related } \\
\text { goals }\end{array}$ & $\begin{array}{l}\text { Type of collaboration } \\
\text { elicited }\end{array}$ & $\begin{array}{l}\text { Type of Interaction } \\
\text { that support the } \\
\text { elicited collaboration }\end{array}$ & $\begin{array}{l}\text { Elements of the } \\
\text { game that support } \\
\text { the elicited } \\
\text { collaboration }\end{array}$ \\
\hline \multirow[b]{2}{*}{1} & Exploration & $\begin{array}{l}\text { Inter-group collaboration } \\
\text { (negotiation) through } \\
\text { physical and verbal } \\
\text { participation }\end{array}$ & $\begin{array}{l}\text { Selection of objects } \\
\text { through cooperative } \\
\text { gesture }\end{array}$ & $\begin{array}{l}\text { Shared workspace } \\
\text { Remote Awareness } \\
\text { Videoconference }\end{array}$ \\
\hline & \multicolumn{4}{|c|}{$\begin{array}{l}\text { In this level, participants explore a collection of ten images distributed on the multi-touch } \\
\text { horizontal surface. Players on both locations must agree and choose an item in order to receive } \\
\text { further information. All members from both groups must simultaneously select the same item } \\
\text { by placing their finger on it (cooperative gesture). Once "all" players simultaneously select an } \\
\text { item, they are presented with additional imagery and a textual description related to the selected } \\
\text { item. The description includes information about the object's origin, functionality, creation } \\
\text { date, and how it became part of the museum collection. }\end{array}$} \\
\hline & $\begin{array}{l}\text { Categorization } \\
\text { of images based } \\
\text { on place of } \\
\text { origin }\end{array}$ & $\begin{array}{l}\text { Inter-and intra-group } \\
\text { collaboration through } \\
\text { physical and verbal } \\
\text { participation. }\end{array}$ & $\begin{array}{l}\text { Simultaneous remote } \\
\text { and co-located } \\
\text { interaction }\end{array}$ & $\begin{array}{l}\text { Shared workspace } \\
\text { Special piece } \\
\text { Remote awareness } \\
\text { Videoconference }\end{array}$ \\
\hline 2 & \multicolumn{4}{|c|}{$\begin{array}{l}\text { In this level, participants must organize the objects according to their country of origin. The } \\
\text { surface table includes a world map with place holders. Students can organize the objects simply } \\
\text { by dragging and placing each object onto the appropriate geographical location. Visual } \\
\text { feedbacks are given in case of correctness of the answer, while visual and auditory hints are } \\
\text { displayed on the vertical screens in case of incorrectness. Each of ten objects can be controlled } \\
\text { independently by holding it or by dragging it, however members from either group can take } \\
\text { control over it only after the item is released. There are two special items, one for each team. } \\
\text { When selected, all others pieces are grayed out and cannot be moved, while on the vertical } \\
\text { screens appear an hint appears and an alert signal is played to call the players to focus on the } \\
\text { hint. Once the piece is correctly located, the others items get released. }\end{array}$} \\
\hline \multirow{2}{*}{3} & $\begin{array}{l}\text { Categorization } \\
\text { of images based } \\
\text { on function } \\
\text { classes }\end{array}$ & $\begin{array}{l}\text { Inter-and intra-group } \\
\text { collaboration through } \\
\text { physical and verbal } \\
\text { participation. }\end{array}$ & $\begin{array}{l}\text { Simultaneous remote } \\
\text { and co-located } \\
\text { interaction }\end{array}$ & $\begin{array}{l}\text { Shared workspace } \\
\text { Special piece } \\
\text { Remote awareness } \\
\text { Videoconference }\end{array}$ \\
\hline & \multicolumn{4}{|c|}{$\begin{array}{l}\text { This level is conceptually identical to the second one. However, on this stage players must } \\
\text { organize items based on their functionality (religious, popular, or entertainment). The type of } \\
\text { interaction and collaboration allowed are identical to the second level. Two special items are } \\
\text { also presented and behave as described above. }\end{array}$} \\
\hline
\end{tabular}




\section{Evaluation}

We conducted a user study to explore the efficiency of the design criteria in promoting collaboration and discussion among participants. The user study provided quantitative and qualitative data to assess how the collaboration features in game lead to different collaborative behaviors, and to assess user's perception and satisfaction with the game.

Table 2. User study's units of analysis and coding scheme for physical and verbal intra-group and inter-group collaboration.

\begin{tabular}{|c|c|c|c|}
\hline Goal & $\begin{array}{l}\text { Unit of } \\
\text { analysis }\end{array}$ & $\begin{array}{l}\text { INTRA-GROUP } \\
\text { definition }\end{array}$ & $\begin{array}{l}\text { INTER-GROUP } \\
\text { definition }\end{array}$ \\
\hline \multicolumn{4}{|c|}{ Quantitative analysis through video coding } \\
\hline Collaboration & $\begin{array}{l}\text { Time in } \\
\text { seconds }\end{array}$ & $\begin{array}{l}\text { Physical: helping moving a } \\
\text { piece, or touching the surface } \\
\text { to indicate the place where to } \\
\text { move the piece } \\
\text { Verbal: suggesting, verbally, } \\
\text { the place where to move the } \\
\text { piece }\end{array}$ & $\begin{array}{l}\text { Physical: touching the surface to } \\
\text { indicate the place where to } \\
\text { move the piece; (in Level 1, } \\
\text { touching the surface to indicate } \\
\text { the item they want to select). } \\
\text { Verbal: suggesting, verbally } \\
\text { through videoconference, the } \\
\text { place where to move the piece; } \\
\text { (in Level 1, suggesting through } \\
\text { videoconference the item to } \\
\text { select). }\end{array}$ \\
\hline $\begin{array}{l}\text { Equity of } \\
\text { Collaboration }\end{array}$ & $\begin{array}{l}\text { Gini } \\
\text { coefficient }\end{array}$ & --- & $\begin{array}{l}\text { Takes the collaboration rate for } \\
\text { each group (physical \& verbal), } \\
\text { and computes the Gini coef. } \\
\text { btw. two groups. }\end{array}$ \\
\hline \multicolumn{4}{|c|}{ Qualitative analysis through questionnaire } \\
\hline $\begin{array}{l}\text { Perceived } \\
\text { Communication }\end{array}$ & Likert scale & $\begin{array}{l}2 \text { questions assessing how } \\
\text { much participants perceived } \\
\text { having communicated with } \\
\text { their co-located partners }\end{array}$ & $\begin{array}{l}2 \text { questions assessing how much } \\
\text { participants perceived to have } \\
\text { communicated with their remote } \\
\text { partners }\end{array}$ \\
\hline $\begin{array}{l}\text { Perceived } \\
\text { Collaboration }\end{array}$ & Likert scale & $\begin{array}{l}\text { Idem perceived } \\
\text { communication }\end{array}$ & $\begin{array}{l}2 \text { questions assessing how much } \\
\text { participants perceived to have } \\
\text { collaborated with their co-locate } \\
\text { partners }\end{array}$ \\
\hline $\begin{array}{l}\text { Overall } \\
\text { satisfaction }\end{array}$ & $\begin{array}{l}\text { Open } \\
\text { question }\end{array}$ & \multicolumn{2}{|c|}{$\begin{array}{l}\text { List } 3 \text { aspects of the experience that you liked/enjoyed } \\
\text { List } 3 \text { aspects of the experience that you didn't like. }\end{array}$} \\
\hline
\end{tabular}

Collaboration and equity

We are interested in exploring three aspects of collaboration: 1) to what extent the game promoted inter- and intra-group collaboration among the different stages of the game, 2) the existence of a preferred type of collaboration, i.e. a tendency of users in collaborating more with the co-located members (intra-group collaboration) than with the remote ones (inter-group collaboration), and 3) to what extent the game promoted 
equal inter-group participation, i.e. if both groups equally collaborated with the other team or if the process of collaboration was mostly initiated by one of the two teams.

We measured both physical and verbal intra-group and inter-group collaboration by identifying the main patterns of participants' interactions and representing them through scores computed as described in table 2 . The unit of analysis for group collaboration was defined as the sum of individual scores coming from the members of the same group. We used the Gini Coefficient to express the equitable nature of collaboration among remote teams. It uses the contribution of each group to compute the deviation with respect of equal participation between the two groups. The group contributions for calculating the Gini coefficient have been computed as described in table 2 .

\section{Overall Satisfaction}

We collected information about overall user satisfaction using a follow-up questionnaire divided into four main categories based on the following criteria: perceived inter-and intra-group communication, perceived inter- and intra-group collaboration, overall satisfaction of the experience. Overall satisfaction was assessed using close and open qualitative questions to elicit positive and negative aspects of four main categories related to aspects of the game, the videoconference, the social nature of the game (e.g. collaboration), the interaction, and issues regarding the installation.

\subsection{Method}

The evaluation consisted of 10 one-shot experimental sessions in which 20 groups (with 2 to 3 persons per group) of 39 first-year university students (14 women, 25 men) played all game levels (lasting on avg. 5.4 min.) in a controlled environment. The stations were set up in two remote rooms at a university campus. Participants in each room were given few minutes to familiarize with the multi-touch table and to introduce themselves to the remote team through videoconference while one researcher explained the main goal of the game to both teams. The researchers remained in the room throughout the session, but did not interact with participants unless in response to specific problems related to technical difficulties. All sessions were video recorded and the actions of each participant were analyzed via video coding using the scheme represented in table 2 .

\subsection{Results}

\section{Collaboration}

Analysis of collaborative patterns in users' activities showed that collaborative engagement of participants decreased as the game progressed: on average, participants collaborated for $23.3 \%, 16.7 \%$, and $13.5 \%$ of the total time for level one, two and three respectively. Further analysis on second and third levels, in which both 
inter and intra-group collaboration are elicited, showed a significant difference $(\mathrm{t}=9.65, \mathrm{p}<0.001)$ in the time spent collaborating in the two modalities (intra-group: $77.8 \%$, Vs. inter-group: $22.2 \%$ ). This indicates that participants were more engaged in talking and interacting with the members of the same team than in discussing with the remote team.

During the entire game, users preferred verbal communication as channel for collaboration rather than physical interaction (e.g. deictic gestures) on the surface. This is particularly true for the inter-group collaboration in level 2 and 3. However, in level 1 , in which cooperative interaction is required (see Table 1), participants used more deictic gestures to communicate with the remote partners with respect to the other levels. This supports the fact that in cooperative manipulation, where the actions of remote users must be strictly coordinated, is particular crucial to support the interaction with elements that provide remote awareness. Additionally, we observed a learning curve regarding simultaneous interactions; on the second stage participants exhibited a turn-taking behavior, while on the $3^{\text {rd }}$ level they interacted with the objects simultaneously and independently. Participants went from an average of 5.2 times giving turns on the second level to only 2.3 times on the third level.

Table 3. Averages of time passed by each group in intra and inter group collaboration.

\begin{tabular}{|c|ccc|crr|c|}
\hline \multirow{2}{*}{ Level } & \multicolumn{2}{|c|}{ Intra-group Collaboration** } & \multicolumn{3}{|c|}{ Inter-group Collaboration** } & Gini Coefficient \\
& Total & Physical & Verbal & Total & Physical & Verbal & \\
\hline 1 & - & - & - & 100 & 45.2 & 54.8 & .52 \\
\hline 2 & 74 & 35.1 & 64.9 & 26 & 13.9 & 86.1 & .25 \\
\hline 3 & 81.9 & 34.7 & 65.3 & 18.1 & 8.5 & 91.5 & .28 \\
\hline
\end{tabular}

** The values represent percentage respect to the total time spent collaborating.

Regarding perceived communication and collaboration, participants scored significantly higher on the perceived intra-group communication (M. 3.1) than the inter-group (M. 2.1), $(\mathrm{t}=3.99, \mathrm{p}<.001)$. Also on the level of collaboration, participants perceived to have collaborating more with the members of the same team (M. 3.13), rather than with the other team (M. 2.4) $(\mathrm{t}=3.42$, $\mathrm{p}<0.001)$, confirming the results of the quantitative analysis.

\section{Equity of collaboration}

Analysis of Gini coefficient revealed differences regarding equity of inter-group collaboration. In the first level one group mostly predominated the task deciding verbally which item to select, while on the others levels both groups initiated collaboration with the remote team, almost equally (.52 vs. .25 and .28).

\section{Overall Satisfaction, Positive and negative Aspects}

The follow-up questionnaire showed that the overall satisfaction of the experience was high (M. 3.8, SD. 0.66, in a scale from 1 to 5). Responses about the positive aspects indicate that participants appreciated the collaborative nature of the game and the type of interaction allowed by the multi-touch interface. Whereas aspects related with the videoconference and general features of the game (such as its intuitiveness, the educative role etc.) scored low values (Fig. 2). Analysis of the negative aspects of 
the game revealed several problems, such as the lack of clarity in the goal of the game, the lack of competition, and the game subject. Many negative complains addressed the audio quality of the videoconference that caused difficulty in the communication with the remote team.
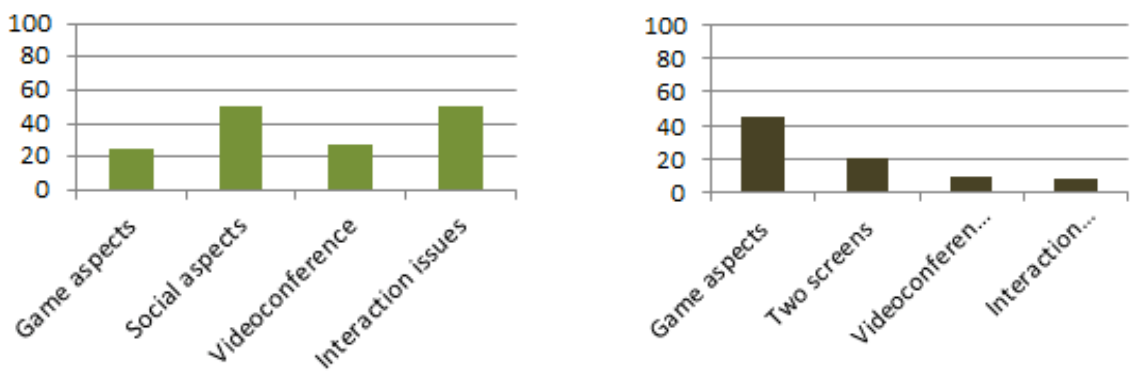

Fig. 2. Positive (left) and negative (right) aspects according to users' opinions: participants appreciated the social collaborative nature of the game and the multi-touch interface, found the split-screen setup uncomfortable and suggested several game improvements.

\subsection{Discussion}

The results indicate that the different stages of the game promoted inter- and intragroup collaboration as participants collaborated up to $23 \%$ of the time. There was a tendency of users in collaborating intra-group as participants spent around $80 \%$ of the time collaborating with their peers. This is partially due to the bad choice of using a two-screen setup; one vertical and one horizontal, as many users did no looked at the vertical videoconferencing screen while interacting on the horizontal screen. This is also supported by the responses about negative aspects of the experience, as many users complained for the lack of affordance of the videoconference while interacting. The results also indicate that both groups collaborated equally in collaborative tasks (levels 2 and 3), while cooperative tasks (level 1) elicited a predominated behavior by one of the two teams.

Despite problems reported regarding the videoconference video quality, the audio link provided a natural way for learners to communicate with remote participants. Participants preferred verbal communication as channel for collaboration rather than physical interaction on the surface. This was especially true for tasks not requiring cooperative actions, as in this case users needed visual cues of remote actions to coordinate the movement with the remote partners. Although our system supports simple cooperative manipulation, i.e. selection of items, the findings suggest that this kind of interaction promotes a higher level of collaboration, but might lead to unequal participation among remote partners.

Participants reported several deficiencies in the game design that should be addressed in future iterations: the lack of a clear goal, competition, and intuitiveness. Another important drawback is that the game can only be used when there is at least one participant on each remote location. This drawback can be easily addressed by 
offering single and collaborative modes of interaction in future iterations. Overall, the results indicate that participants appreciated the collaborative nature of the game and the type of interaction allowed by the multi-touch interface.

\section{Conclusions and Future Work}

This paper has introduced a customizable system used to develop collaborative informal learning experiences in remote museum-like environments. An interactive learning experience combines multi-touch interaction and videoconferencing to engage students in multi-user collaborative problem solving activities. The system facilitates remote collaboration allowing participants to communicate through videoconferencing, in a vertical screen, and through a shared interactive workspace displayed on a horizontal screen.

The findings assessing the game features show that the system promoted different collaborative behaviors and enabled inter- and intra-group collaboration and cooperation. Participants collaborated up to $23 \%$ of the time with a tendency for intragroup collaboration. Moreover, remote groups collaborated equally in collaborative tasks while collaboration was mostly initiated by one of the two teams for the cooperative tasks. Finally, user's perception and satisfaction was positive as participants appreciated the collaborative nature of the game, the type of interaction allowed by the multi-touch interface, and the videoconference communication channel. To conclude, our findings lend support to the view that augmenting videoconferencing systems with a tangible shared space offers new possibilities and scenarios for remote collaborative environments and collaborative learning.

Future work includes addressing the design problems encountered; improving the intuitiveness of the interface, adding competition aspects to the game, conducting new experiences in other educational spaces. Moreover, we are currently working on integrating videoconferencing within a $3 \mathrm{D}$ collaborative-shared space to support a more ergonomic interaction between remote participants, allowing for eye contact while interacting with the surface at the same time [1].

Acknowledgments. This work has been partially funded by the LEARN 3 project (TIN2008-05163/TSI) and by the European Fund for Regional Development as part of the Catalonian FEDER 2007-2013 project.

\section{References}

1. Arroyo, E., Ardaiz, O., Righi, V., Blat, J.: Distributed Multi-touch Virtual Collaborative Environments. In: Proceedings of CTS 2010, Illinois, USA, pp. 635-636, (2010).

2. Anastasiades,P.S., Vitalaki, E., Gertzakis,N... Collaborative learning activities at a distance via interactive videoconferencing in elementary schools: Parents' attitudes. Computers \& Education, vol. 50, pp. 1527-1539, (2008). 
3. Bachour K., Kaplan F., Dillenbourg P.: An interactive Table for Supporting Participation Balance in Face-to-Face Collaborative Learning. In: IEEE Transactions on Learning Technologies, 3(3), 203--213, (2010).

4. Baker,M. \& Lund, K.: Promoting reflective interactions in a CSCL environment. Journal of Computer Assisted Learning, 13, (3), (2003).

5. Brereton, M. and McGarry, B.: An observational study of how objects support engineering design thinking and communication: implications for the design of tangible media. In: Proceedings of SIGCHI '00. ACM, New York, NY, USA, 217-224, (2000).

6. Ellis, C.A., Gibbs J.B., Rein, G.: Groupware: some issues and experiences. Commun . ACM 34, 1 (January 1991), 39-58. (1991).

7. Harris, A., Rick, J., Bonnett, V., Yuill, N., Fleck, R., Marshall, P. and Rogers, Y. Around the table: Are multiple-touch surfaces better than single-touch for children's collaborative interactions? In Proceedings of CSCL 2009, p.p 335-344. ISLS, (2009).

8. Patrick Jermann, Guillaume Zufferey, et al: Tinkering or Sketching: Apprentices' Use of Tangibles and Drawings to Solve Design Problems. EC-TEL 08, pp. 67-178, (2008).

9. Joan I Rosa Josep. La col·lecció de col·leccions de Guy Selz. ACTAR, Arts Santa Monica, Barcelona (2010).

10. Kemp R., Kemp E., Mohanarajah T.: Supporting Group Learning Using a Digital Whiteboard, M. Masoodian et al. (Eds.): APCHI, LNCS 3101, pp. 594--598 (2004).

11. Lewis G. J., Hasan S. M., Alexandrov, et al: Collaborative Virtual Environment for Advanced Computing. Computers and Artificial Intelligence, 24(1), (2005).

12. Looi, C-K.,Chen, W. And Ng, F-K: Collaborative activities enabled by GroupScribbles: An exploratory study of learning effectiveness. Comp. \& Ed. 54 (1), pp 14--26, (2010).

13. Margery D. M., Arnaldi B., Plouzeau N.: A general framework for cooperative manipulation in virtual environments. In: Proceedings of Virtual Environments '99. Springer (pp 169--178), New York, (1999).

14. Moore, M.: Three types of interaction. The American J. of Distance Ed., 3, 1-7, (1989).

15. Panagiotes S. Anastasiades*, Elena Vitalaki, Nikos Gertzakis.:Collaborative learning activities at a distance via interactive videoconferencing in elementary schools: Parents' attitudes. Computers \& Education, vol. 50, pp. 1527-1539, (2008)

16. Pauchet, A., Coldefy, F., Lefebvre, L., et al..: Tabletops: Worthwhile Experience of Collocated and Remote Collaboration. Proc. TABLETOP 2007, 27--34, (2007).

17. Pawar, U., Pal, J., \& Toyoma, K. Multiple Mice for Computers in Education in Developing Countries. In: ICTD '06, pp. 64-71, (2006).

18. Pintrich, P. R. A motivational science perspective on the role of student motivation in learning and teaching contexts. J. of Educational. Psychology, 95(4), 667-686, (2003).

19. Roschelle, J.: GroupScribbles: A tool for highly interactive Tablet PC classrooms. In: HP Webinar on Teaching, Learning, \& Technology in Higher Ed, (2006).

20. Schenker, J. L.: Economic woes boost videoconferencing. Bloomberg Businessweek, (2008). Retrieved from http://www.businessweek.com/

21. Stanton D., Neale H.R.: The effects of multiple mice on children's talk and interaction. Journal of Computer Assisted Learning, 19(2). p. 220-228, (2003).

22. Stefik M., Bobrow D.G., et al. WYSIWIS revised: early experiences with multi-users interfaces. In: ACM Trans. Inf. Syst., Vol. 5, No. 2. pp. 147-167, (1987).

23. Tang, A., Neustaedter, C. and Greenberg, S.: VideoArms: Embodiments for Mixed Presence Groupware. In: BCS-HCI (2006)

24. Tuddenham, P., and Robinson, P.: Distributed Tabletops: Supporting Remote and MixedPresence Tabletop Collaboration. In: TABLETOP'07. pp. 19-26, (2007).

25. Vom Lehn D., Heath C., et al: Exhibiting Interaction: Conduct and Collaboration in Museums and Galleries. Symbolic Interaction J. Vol. 24, No. 2, pp. 189-216, (2001). 